\title{
Characterization of underground rock masses employing structure from motion: Application to a real case
}

\author{
R. García-Luna, S. Senent, R. Jurado-Piña \& R. Jimenez \\ Technical University of Madrid, Madrid, Spain
}

\begin{abstract}
We propose an inexpensive and easy-to-use methodology for remote characterization of underground rock masses at the tunnel face. The method is based on the joint use of the Structure from Motion (SfM) photogrammetric technique and a Discontinuity Set Extractor (DSE) software, in which a high-density 3D point cloud is created using a commercial SfM software (Agisoft PhotoScan). To maintain the real features of the tunnel face, such digital model must be orientated and scaled; therefore, a "portable orientation template" is employed. The point cloud is then introduced in DSE, where discontinuity orientation data are "extracted" and analyzed to identify joint sets. The measurements obtained with the SfM + DSE approach have been compared with those obtained with a traditional analysis based on manual compass measurements. Both techniques identify the same number of discontinuity sets with orientation differences within the uncertainty range associated to manual measurements.
\end{abstract}

\section{INTRODUCTION}

A good knowledge of the discontinuity network in a rock mass is fundamental for the design and control of tunnels under construction, as discontinuities are surfaces of weakness that control the occurrence of unstable blocks in the tunnel and the loads imposed by the blocks on the excavation support (Goodman 1976, Hudson \& Harrison 1997). However, characterizing these discontinuities using manual methods (i.e. direct measurements made with compass) can impose a risk on the safety of the operators who take the data, as the (unsupported) tunnel face is the area of the tunnel with a higher risk of collapse (Slama et al. 1980, Health and Safety Executive 1996). In addition, the results obtained manually are often very subjective, depending on factors such as the access conditions to the outcrop, the number of measurements made, the worker's ability, the time available and the scale of the problem.

Due to these difficulties, remote "non-contact" techniques — such as digital photogrammetry and laser scanner methods - are becoming increasingly common to obtain digital 3D models of tunnel supports (Roncella et al. 2012, Chaiyasarn et al. 2015) and to identify and characterize the underground rock mass discontinuities (Buyer \& Schubert 2016, 2017). However, these works do not discuss in detail the performance of the remote technique.

In this paper we introduce an inexpensive and easy-to-use methodology for remote identification of discontinuity sets at underground rock masses under real tunneling conditions. The proposed methodology has been applied to one tunnel under construction in Northern Spain, comparing the results obtained using the remote SfM technique with those obtained using traditional data collection methods with a compass, and describing the photographic conditions for acquisition of the 3D digital model, emphasizing the importance of using auxiliary processing tools that allow us to visualize jointly the main discontinuity sets identified by DSE and the real tunnel scenario as a $3 \mathrm{D}$ point cloud. 


\section{METHODOLOGY}

This process employed to introduce and validate the methodology is divided into three parts. First, we characterize the main discontinuities sets present in the tunnel face using traditional manual measurements (with compass) and a DIPS analysis (Rocscience 2017). The next step is to generate a photogrammetric 3D model from several overlapping photographs of the tunnel face with the commercial software Agisoft PhotoScan (LLC 2016), and using the structure from motion technique combined with a portable orientation template to orientate and scale the model. Then once the 3D model is ready, it is introduced in the open-source software DSE (Riquelme et al. 2014), where the different discontinuity sets data are "extracted" and analyzed. Finally, the results provided by both methods are validated through two different ways: (i) comparing the results obtained with DIPS (in terms of Dipdir/Dip) directly with the measurements obtained with the SfM + DSE approach, and (ii) making a combined 3D visual inspection of the point cloud classified with DSE in superposition with the original unclassified point cloud.

\subsection{Portable orientation template}

One of the main disadvantages of the SfM photogrammetric technique, in contrast to traditional photogrammetry, is that it generates digital 3D models within an arbitrary reference system without scale or orientation. Therefore, to geo-reference the 3D model, it is necessary to include several ground control points (GCPs) of known 3D coordinates (x-y-z), within the scene. Singularities or traces easily recognizable in the images, may also be useful for this purpose (Dowling et al. 2009, Dandois \& Ellis 2010).

To solve this problem, we have developed a "portable orientation template" that avoids the need to use topographic control devices (Figure 1). Its operation is inspired by a conventional compass, but on a larger scale. The template contains five GCPs labeled inside a rigid square built with EVA foam; a central one that serves as the origin of the system of coordinates (local) and other four located at the corners. The template incorporates an arrow that can be aligned with the north using a compass and a spirit level can be used to place it horizontally. As the distance between the points is known, the template serves as reference plane and we can use it to orientate and scale the scene in a single step, using the relative coordinates provided by its points, within a local reference system.

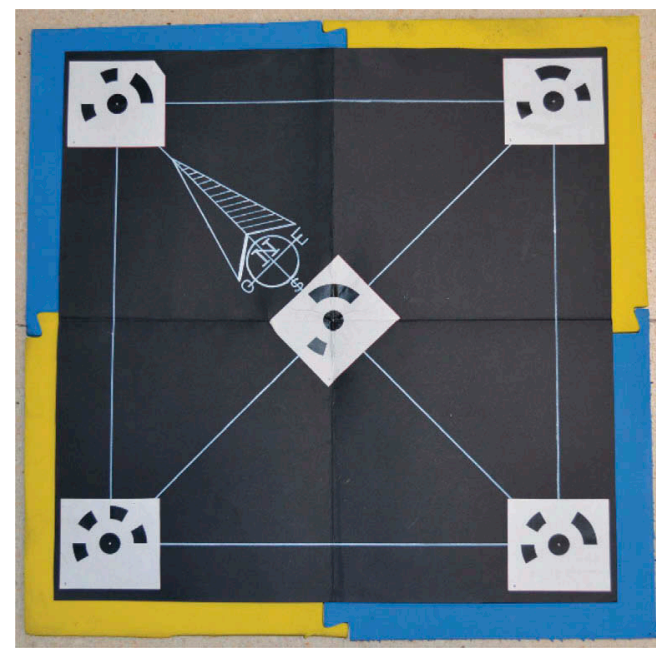

Figure 1. Portable orientation template. 


\subsection{Description of the tunnel}

The methodology has been tested in a real tunnel under construction in the Santa Barbara Foundation research area (FSB 2017), located in Northern Spain. The rock mass is constituted by limolites, lutites and sandstones (RMR 40-60), and the tunnel had an excavated cross-section of $60 \mathrm{~m} 2$ and a length of approximately $295 \mathrm{~m}$ when this study was conducted. The tunnel is constructed using drill and blast methods, with full-face excavation and it is supported using rock bolts and shotcrete. Shotcrete was present in the lower parts of the tunnel face, hiding the rock mass surface and obstructing the real discontinuities.

\subsection{Manual characterization of discontinuity sets}

With the purpose of validating and comparing the results provided by the SfM + DSE methodology, we decided to identify first the main discontinuity sets manually using a traditional compass measurements and a DIPS analysis. We measured the orientations of 33 discontinuities found at the tunnel face with a Freiberger-type geological compass, taking about 30 minutes to complete and register all the measurements. Figure 2 shows the results provided by DIPS (pole density) and the three main discontinuity sets identified using such measurements.

The statistical analysis of the pole vectors of all discontinuities measured with the compass (Figure 2) identifies three main discontinuity sets: F1 (240/79), F2 (131/71) and F3 (4/10). The two first (F1-F2) outcrop clearly at the tunnel face, being cut at $45^{\circ}$ by a N-S vertical sampling surface (note that the direction of advance of the tunnel at the time of data collection was W-E). Both families $(\mathrm{F} 1-\mathrm{F} 2)$ present conjugate orientations $\left(\Delta\right.$ Dipdir $\left.=109^{\circ}\right)$ with sub-vertical dip angles $\left(\beta=79-71^{\circ}\right)$. The last family (F3) presents a sub-horizontal dip angle $\left(\beta=10^{\circ}\right)$ and a more variable dip direction $\left(\alpha=12-273^{\circ}\right)$. The orientation of F3 family, combined with the orientation of the tunnel face, make this discontinuity set to accumulate shotcrete, especially in the lower parts of the tunnel face. This complicates the measurement of F3 orientations, which are better measured at reverse faces (wall), which only appear clearly in the upper area of the face, inaccessible for manual measurements.

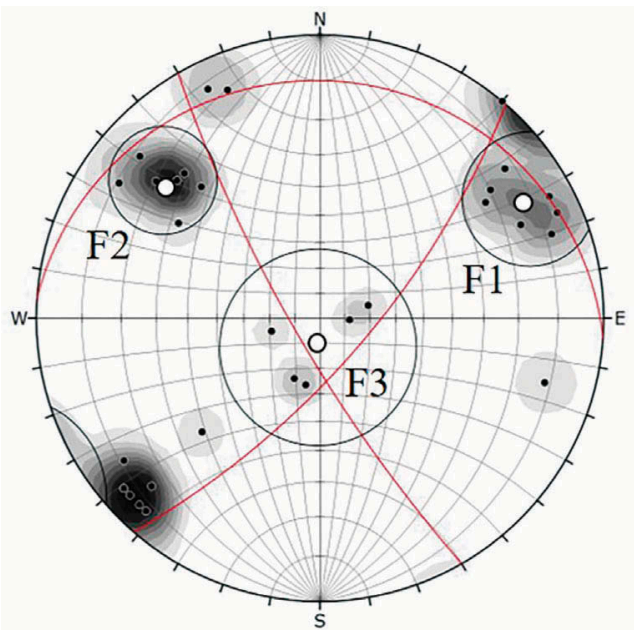

Figure 2. Stereographic projection (lower hemisphere, equal angle) of the pole vectors of discontinuity orientations measured with the compass (black dots) and the three main discontinuity sets (F1-F2-F3) identified by DIPS (white dots). 


\subsection{Photographic equipment and data acquisition}

To generate the photogrammetric 3D model, we employed an un-calibrated Sony Digital Single-Lens Reflex camera (model $\alpha 230$ ) with a common lens Sony DT 18-55 mm (F 3.5-5.6). There are some challenges for 3D SfM reconstruction in underground spaces, such as the need to work in low-luminosity conditions while maintaining low exposition times (of $<1 / 4$ ") to avoid the effect of micro-vibrations in the field. To overcome them, we decided to install the camera on a professional heavy weigh tripod (model Yashica YT-300) and to activate the camera by remote control.

The same lighting conditions as in a real tunnel under construction have been used in this work. The tunnel face was illuminated by a drilling jumbo positioned $4 \mathrm{~m}$ away from the face, which provided a luminosity of about 100-130 LUX (measured at the face). In addition, we use an extra portable illumination source situated directly above the camera (LED torch, model Yongnuo YN-216 LEDs) using an L-Bracket plate on the tripod. This torch provided an extra illumination of the rock surface of about 10-30 LUX (depending on the distance from the tunnel face) that pointed directly at the same direction of the camera, helping us to attenuate the presence of "shadow areas" that can produce occlusions zones in the final 3D model.

We make a full-scan from the tunnel face, taking 206 photographs from 18 different positions (Figure 3), achieving a high level of superposition between each picture ( $>60 \%)$. All the photographs were taken with the highest quality settings available in the camera (10 MP, RAW-JPEG format) and with fixed camera adjustments (manual mode, AF, focal length 18 $\mathrm{mm}$, aperture $\mathrm{F} / 3.5$, shutter speed $1 / 4$ " and ISO 100). These parameters are defined before photo shooting according to the available lighting conditions of the tunnel face; in this way, the number of maneuvers between photographs is minimized (point-stabilize-shoot), helping us to streamline the process of data collection.

In order to be able to geo-reference and scale the 3D model correctly, the portable orientation template was located directly on the ground (oriented and levelled), at the lower part of the tunnel face before starting the shooting. In addition, seven precision targets were distributed along the surface of the tunnel face to evaluate the accuracy of the scale of the 3D model provided by the portable orientation template (comparing the distances between them, measured "in situ" with measuring tape with the corresponding distances computed from the scaled 3D model).

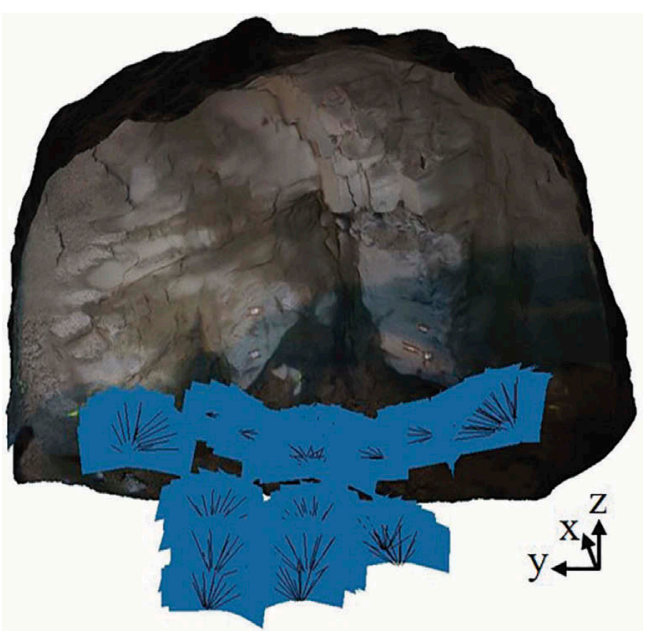

Figure 3. Front view of the photogrammetric 3D model (textured mesh) and camera positions (blue squares). 
The total time spent in the preparation and acquisition of photogrammetric data was 30 minutes, or about the same time employed in the field campaign for the manual characterization with compass. Although these times may seem very high, it was decided to perform an over-characterization of the tunnel face to ensure the generation of a solid 3D model.

\subsection{Generation of the 3D model}

The 3D model was constructed with the professional version of Agisoft PhotoScan photogrammetric software, using the structure from motion approach. (Note that the standard version does not allow to work with reference points.) The surface of the whole 3D model has a total area of $192 \mathrm{~m} 2$ and was generated from all the available photographs (206). The total number of points was $6,058,037$, with a density of 2.5 points $/ \mathrm{cm} 2$. The resulting 3D point cloud was finally scaled and oriented, according to a local reference system, based on the five GCPs included within the portable orientation template. The errors of the relative coordinates $(x-y-z)$ of the reference points provided by the template are listed in Table 1. Table 2 summarizes the distance errors for precision targets (7) installed on the tunnel face.

Generation the photogrammetric 3D model in Agisoft PhotoScan took a computation time of about $25 \mathrm{~h}$, including the time to align and orient the initial "disperse" point cloud (1h 46 $\mathrm{min}$ ) and the time to process the second high-density point cloud ( $23 \mathrm{~h} 45 \mathrm{~min}$ ) using MVS techniques. (All computations were performed using a single core of an Intel Core i7-6700 computer [3.4GHz processor, $16 \mathrm{~GB}$ RAM] running the Windows operating system.)

Finally, so that the results are not affected by the presence of shotcrete in the tunnel, the zones of the model where its presence is greater have been eliminated, keeping only the central area of the front, where the rock mass is not covered with shotcrete. In addition, an automatic filtering (subsample) of the point cloud was conducted, establishing a minimum distance of $1 \mathrm{~cm}$ between the points, in order to avoid that the areas of the model with higher density (points $/ \mathrm{cm} 2$ ) can affect the DSE analysis (Riquelme 2015). Both the edition of the cloud and the subsampling have been performed with the 3D CloudCompare software (Girardeau-Montaut 2016.). In this way the final "Region of interest" of the model (ROI), with a total of 295,705 points, is defined.

Table 1. Errors of $(x-y-z)$ coordinates of template's reference points within the $3 \mathrm{D}$ model.

\begin{tabular}{llll}
\hline GCPs & $\mathrm{x}$ & $\mathrm{y}$ & $\mathrm{z}$ \\
\cline { 2 - 3 } & $\mathrm{mm}$ & $\mathrm{mm}$ & $\frac{\mathrm{mm}}{\mathrm{mm}}$ \\
\hline 1 & -2.8 & 1.4 & 1.7 \\
2 & -3.8 & 3.1 & 2.5 \\
3 & -0.3 & 0.3 & 1.9 \\
4 & -3.8 & -2.5 & 2.1 \\
5 & -6.7 & 0.4 & 2.4 \\
\hline
\end{tabular}

Table 2. Distance errors for precision targets.

\begin{tabular}{|c|c|c|c|}
\hline \multirow[t]{2}{*}{ Scale Bars } & Real distance* & 3D distance & Error \\
\hline & $\mathrm{mm}$ & $\mathrm{mm}$ & $\mathrm{mm}$ \\
\hline target $1-$ target 2 & 441 & 451.0 & 10.0 \\
\hline target $3-$ target 4 & 755 & 776.0 & 21.0 \\
\hline target $5-$ target 6 & 547 & 561.1 & 14.1 \\
\hline target $5-$ target 7 & 598 & 611.7 & 13.7 \\
\hline target $6-$ target 7 & 350 & 359.1 & 9.1 \\
\hline
\end{tabular}

*Measured with tape. 


\section{RESULTS AND DISCUSSION}

The study was developed in two simultaneous phases: (i) an initial phase in which the 3D model was analyzed in its entirety, validating the manual results (identifying F1, F2 and F3); and (ii) a secondary phase that focused on a smaller upper sector of the tunnel, resulting in the characterization of a new accessory family, associated to F2 and named F2B, which had not been detected in the initial analysis of manual measurements with DIPS. This case has also been analyzed in more detail in García-Luna et al. (2019).

\subsection{Identification of discontinuity sets with DSE}

Figure 4 and Table 3 show the results provided by the Discontinuity Set Extractor analysis of the high-density point cloud of the ROI (295,705 points) generated by Agisoft PhotoScan and subsampled by CloudCompare. In particular, Figure 4 shows a lower-hemisphere equal-angle stereological projection of the density deviations of the plotted points normal vectors recognized by DSE using all planes identified within the ROI. Table 3 summarizes the representative orientations values (in terms of Dipdir/Dip) of the principal poles of each identified discontinuity set. In total 117,026 points were classified and 178,679 points have not been assigned.

Altogether, three main discontinuity sets were identified with DSE: F1 (249/82), F2 (132/73) and F3 (352/19). Note that they are approximately equal to those identified with DIPS using the compass measurements: two sub-vertical and conjugated discontinuities (F1-F2, with $\Delta$ Dipdir $=117^{\circ}$ ) and one sub-horizontal $(\mathrm{F} 3)$, with maximum differences between their

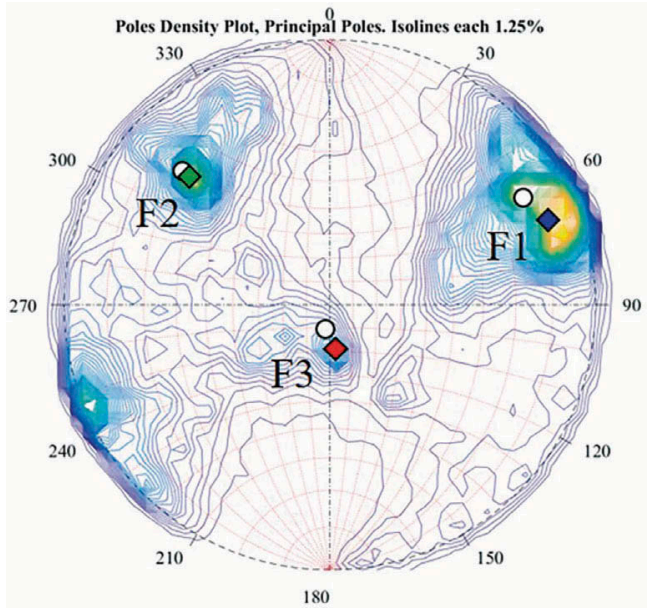

Figure 4. Stereographic projection (lower hemisphere, equal angle) of the plotted points normal vectors (density deviations) and the three main discontinuity sets (F1-F2-F3) represented with diamond symbols, both calculated by DSE. Results of the manual characterization with compass are also provided (white dots).

Table 3. Representative orientations provided by DSE.

\begin{tabular}{|c|c|c|c|}
\hline \multirow[t]{2}{*}{ Discontinuity sets } & Dip Dir. & Dip & Number of assigned points* \\
\hline & {$\left[{ }^{\circ}\right]$} & {$\left[{ }^{\circ}\right]$} & [\%] \\
\hline F1 & 249 & 82 & 27.60 \\
\hline $\mathrm{F} 2$ & 132 & 73 & 11.01 \\
\hline F3 & 352 & 19 & 1.95 \\
\hline
\end{tabular}

*Over the total number of points 
Table 4. Differences of orientation between the discontinuity sets identified with DIPS and DSE.

\begin{tabular}{llllllll}
\hline Discontinuity sets & \multicolumn{2}{l}{ DIPS } & & \multicolumn{2}{l}{ DSE } & & Differences \\
\cline { 2 - 3 } & Dip Dir. $\left[^{\circ}\right]$ & Dip [ $\left.{ }^{\circ}\right]$ & & Dip Dir. $\left[^{\circ}\right]$ & Dip [ $\left.{ }^{\circ}\right]$ & & {$\left[{ }^{\circ}\right]$} \\
\hline F1 & 240 & 79 & & 249 & 82 & 9 \\
F2 & 131 & 71 & & 132 & 73 & 2 \\
F3 & 4 & 10 & & 352 & 19 & 9 \\
\hline
\end{tabular}

corresponding orientations of only $9^{\circ}, 2^{\circ}$ and $9^{\circ}$, respectively. Table 4 shows the differences between the orientations computed with both approaches, using the difference between the acute angle formed by their unit normal vectors (Jimenez-Rodriguez \& Sitar 2006).

The angular differences shown in Table 4 are within the error range associated to manual measurements for these types of planes, of about $6-11^{\circ}$ depending on the dip angle of the joint (Robertson 1970, Windsor \& Robertson 1994).

\subsection{Visual inspection of the results and detail analysis}

One main advantage offered by the use of digital 3D models for the analysis of discontinuities is the wide range of modelling tools associated with the SfM technique. For instance, the 3D point cloud processing software CloudCompare allow us to represent the planes associated to each discontinuity set identified with DSE (F1-F2-F3) back into the original 3D model developed with Agisoft PhotoScan (see Figure 5).

Figure 5 shows that joint-sets F1 and F2 were identified clearly along a great percentage of the face, whereas the F3 set appears less often. However, in the upper area of the 3D model (where several blocks produced by the intersections of the discontinuities planes F1, F2 and F3 can be clearly seen), the sub-vertical F2 set is not well represented. This observation introduces doubts about the discontinuity F2 itself and made us to conduct an alternative analysis of that area of the tunnel face. Therefore, we created a new detailed ROI of this region of approximately $20 \mathrm{~m} 2$ (1/3 of the front surface) with 40,509 points.

The results provided by DSE for this detailed area are shown in Figure 6b, where two main families with a NE-SW component are observed: F2A (130/80) and F2B (157/77). The first one corresponds to the same family previously identified as F1 (132/73) with a slight increase in its dip angle $\left(7^{\circ}\right)$, while the $3 \mathrm{D}$ visualization of the F2B planes (Figure 6a) confirms that they represent a "new" discontinuity family. This new family

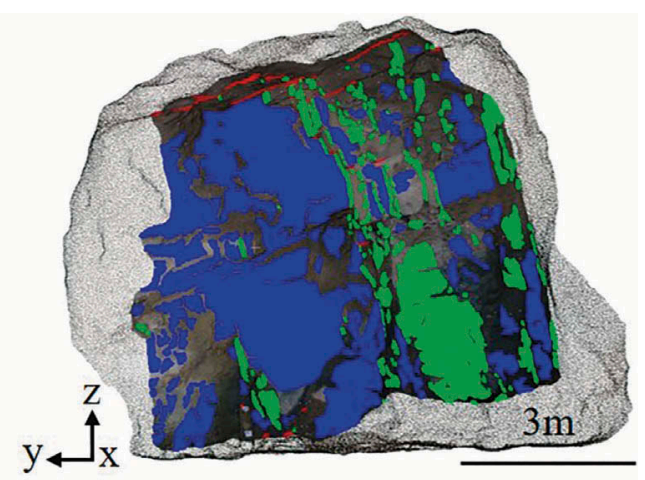

Figure 5. Spatial distribution of surfaces associated to the main discontinuity sets (FI-F2-F3) identified with DSE, projected back into the original 3D model. 

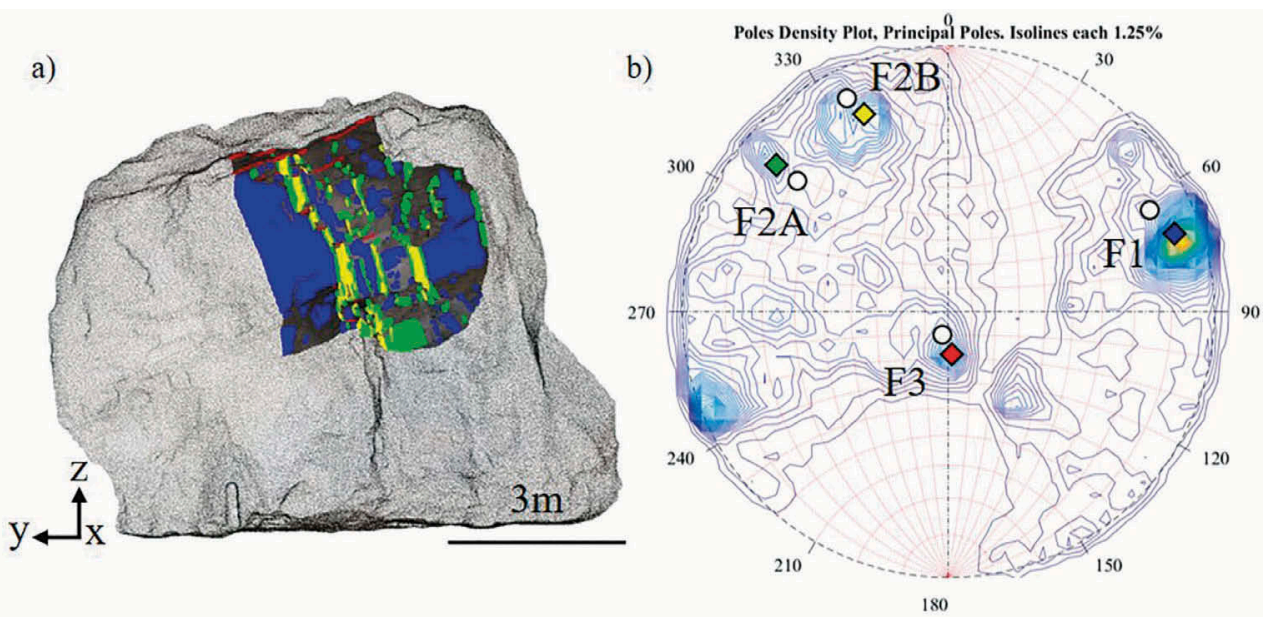

Figure 6. (a) Spatial distribution of surfaces associated to the main discontinuity sets (FI-F2AF2B-F3) identified with DSE, projected back into the new ROI of the upper area. (b) Stereographic projection (lower hemisphere, equal angle) of the plotted points normal vectors (density deviations) and the four main discontinuity sets (F1-F2A-F2B-F3) represented with diamond symbols, both calculated by DSE. Results of the manual characterization with compass are also provided (white dots).

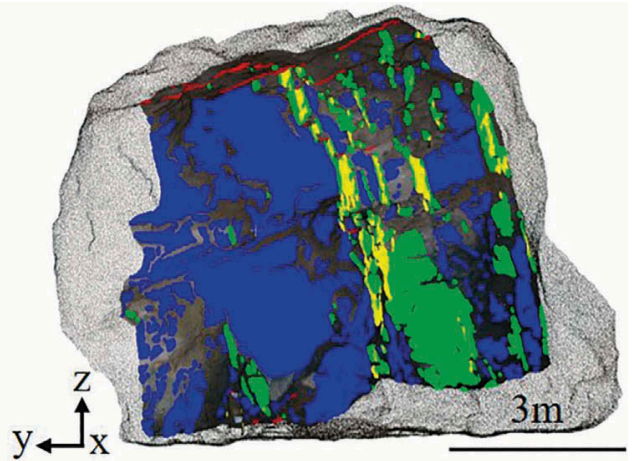

Figure 7. Spatial distribution of surfaces associated to the main discontinuity sets (FI-F2A-F2B-F3) identified with DSE, projected back into the original 3D model.

(F2B) is able to delimit correctly the contour of the blocks for which the first classification had left doubts around the F2 family.

A more detailed analysis of the DIPS results (Figure 2) confirms the presence of this family within the stereonet F2B (156/83), as it was registered in two compass measurements (158/82 and 154/84) that where initially discarded when F2 was characterized.

Once this measure has been fixed it is possible to return to the original model (ROI 295,705 points), to introduce the new discontinuity F2B and to proceed to the joint visualization of the four discontinuity sets: F1, F2A, F2B and F3 (Figure 7). It can be observed that the family F2A (in green) is distributed along the entire face, although it appears more often in the lower areas, where compass measurements are possible $(0-2 \mathrm{~m})$. However, the family F2B (in yellow) mainly appears in the upper area of the tunnel face (unreachable for 
direct measurements) and its presence in the lower parts is rare, which justifies that only two manual measurements of this set were registered.

\section{CONCLUSIONS}

Compared with traditional ground photography, underground characterization of rock masses using digital photogrammetry is challenging due to a dark environment that demands a greater care for shooting preparation, ensuring the stability of the sensor.

In this work, however, has been proved that the generation of 3D models of rock masses in underground environments with real working lighting conditions (provided by drilling jumbos) is possible using a regular camera installed on a tripod and without having advanced photographic knowledge; hence becoming a suitable methodology for real construction projects.

In addition, the use of a portable orientation template has been shown to be a good solution to orientate and scale the model without the need to employ plenty of time and resources.

The analysis performed with the methodology proposed in this paper (SfM + DSE) identifies the same number of families as the analysis performed with traditional methods, showing only slight angular differences that are within the stablished uncertainty range associated to manual compass measurements. Moreover, the use of this technique allows to analyze inaccessible areas of the tunnel face usually not registered by operators (above $0-2 \mathrm{~m}$ ), also becoming a safer worker environment since the hazards associated to collapses are reduced.

Finally, we have proved that the use of alternative tools to filter and fragment the tunnel face into smaller regions is a good solution to focus the analysis on the most important outcrop areas, where the discontinuities surfaces are not hidden by shotcrete accumulation. This process also reduces processing times and decrease the weight of digital files.

\section{REFERENCES}

Buyer, A. \& Schubert, W. 2016. Extraction of discontinuity orientations in point clouds. Rock Mechanics and Rock Engineering: From the Past to the Future 2: 1133-1138.

Buyer, A. \& Schubert, W. 2017. Calculation the Spacing of Discontinuities from 3D Point Clouds. Procedia engineering 191: 270-278.

Chaiyasarn, K., Kim, T., Viola, F., Cipolla, R. \& Soga, K. 2015. Distortion-free image mosaicing for tunnel inspection based on robust cylindrical surface estimation through structure from motion. Journal of Computing in Civil Engineering 30 (3): 04015045.

Dandois, J.P. \& Ellis, E.C. 2010. Remote sensing of vegetation structure using computer vision. Remote Sensing 2 (4): 1157-1176.

Dowling, T., Read, A. \& Gallant, J. 2009. Very high resolution DEM acquisition at low cost using a digital camera and free software 2479-2485.

FSB, Fundación Santa Bárbara. http://www.fsbarbara.com.

García-Luna, R., Senent, S., Jurado-Piña, R. \& Jimenez, R. 2019. Structure from Motion photogrammetry to characterize underground rock masses: experiences from two real tunnels. Tunnelling and Underground Space Technology 83: 262-273.

Girardeau-Montaut, D. 2016. Cloud Compare: 3D point cloud and mesh processing software, opensource project. Version 2.6.

Goodman, R.E. 1976. Methods of Geological Engineering in Discontinuous Rocks. St. Paul: West Publishing Company.

Health and Safety Executive. 1996. Safety of New Australian Tunnelling Method (NATM) Tunnels. A review of sprayed concrete tunnels with particular reference to London clay. Sudbury: (HSE) Books.

Hudson, J.A. \& Harrison, J.P. (1 ed.) 1997. Engineering Rock Mechanics: An Introduction to the Principles. Tarrytown: Pergamon.

Jimenez-Rodriguez, R. \& Sitar, N. 2006. A spectral method for clustering of rock discontinuity sets. International Journal of Rock Mechanics and Mining Sciences 43 (7): 1052-1061.

Llc, A. 2016. Agisoft PhotoScan Professional Edition. Version 1.2. 
Riquelme, A.J., Abellán, A., Tomás, R. \& Jaboyedoff, M. 2014. A new approach for semi-automatic rock mass joints recognition from 3D point clouds. Computers \& Geosciences 68: 38-52.

Riquelme, A.J. 2015. Uso de nubes de puntos 3D para identificación y caracterización de familias de discontinuidades planas en afloramientos rocosos y evaluación de la calidad geomecánica ( $\mathrm{PhD}$ Thesis). Universidad de Alicante.

Rocscience Inc. 2017. Dips User Manual. Version 7.0.

Roncella, R., Umili, G. \& Forlani, G. 2012. A novel image acquisition and processing procedure for fast tunnel DSM production. International Archives of the Photogrammetry, Remote Sensing and Spatial Information Sciences 297-302.

Slama, C.C., Theurer, C. \& Henriksen, S.W. 1980. Manual of Photogrammetry. American Society of photog rammetry. Falls Church, Va., United States. 